\title{
Sultamicillin in the treatment of gonorrhoea caused by penicillin sensitive and penicillinase producing strains of Neisseria gonorrhoeae
}

\author{
W A ATIA,* A M EMMERSON,† AND D HOLMES*
}

From the *Department of Genitourinary Medicine, Royal Northern Hospital, and the +Department of Microbiology, Whittington Hospital, London

SUMMARY A single oral dose of $2 \mathrm{~g}$ of sultamicillin and $1 \mathrm{~g}$ of probenecid was effective in the treatment of men with uncomplicated gonococcal urethritis caused by both penicillin sensitive strains of Neisseria gonorrhoeae and penicillinase producing strains of $N$ gonorrhoeae (PPNG). Of 94 infected men who attended for at least one follow up examination, 91 (97\%) were cured. The remaining three (3\%) patients were still infected at follow up. Two of these patients had been re-exposed to an infected partner and were considered to be possible reinfections, while the third was deemed a treatment failure. Six of the 94 patients were infected with PPNG strains and all were successfully treated. Plasmid analysis of the PPNG strains showed Asian and African types both with and without transfer plasmid.

\section{Introduction}

Sultamicillin (Pfizer Laboratories, CP-49, 952-15) is a novel compound in which ampicillin and the $\beta$-lactamase inhibitor sulbactam (Pfizer Laboratories, CP-45, 899) are linked as a double ester as shown in the figure. During absorption the compound is hydrolysed, delivering both ampicillin and sulbactam to the blood in equivalent molar proportions. Pharmacokinetic studies have shown that sultamicillin provides up to three times higher serum concentrations of ampicillin than would be achieved by giving an equivalent dose of ampicillin alone. ${ }^{1}$ Sulbactam (penicillanic acid sulphone) possesses potent inhibitory activity against a wide variety of both pericytoplasmic and secreted $\beta$-lactamases from Gram positive and Gram negative bacteria and has the advantage of greater stability in aqueous solution than clavulanic acid. ${ }^{2}$ The combination of sulbactam with ampicillin is effective in restoring activity against a range of ampicillin resistant bacteria including $\beta$-lactamase producing strains of Neisseria gonorrhoeae. In view of the increasing prevalence (nationally ${ }^{3}$ and world wide) of penicillinase producing strains of $N$ gonorrhoeae

Address for reprints: Dr A M Emmerson, Department of Microbiology, Whittington Hospital, St Mary's Wing, Highgate Hill, London N19 5NF

Accepted for publication 13 March 1983

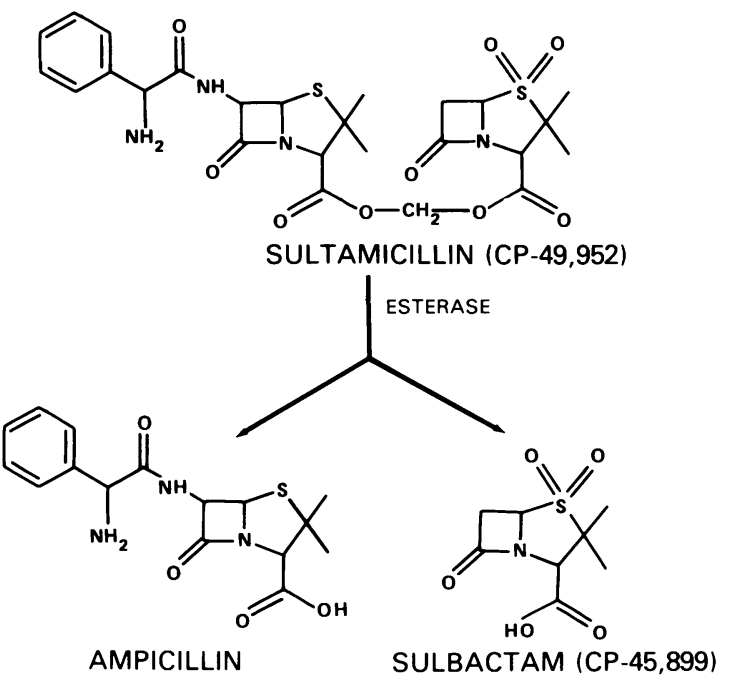

FIGURE Chemical composition of sultamicillin showing its relation to ampicillin and sulbactam.

(PPNG) we decided to study the efficacy of sultamicillin in gonorrhoea.

\section{Patients and methods}

STUDY POPULATION

One hundred and thirteen men with uncomplicated gonococcal urethritis initially diagnosed by micro- 
scopy of Gram stained smears of urethral discharge were included in the trial having given informed consent. Patients known to be allergic to penicillins or cephalosporins or who were receiving other investigational drugs or antibiotic treatment were excluded. Patients were also excluded at first interview if attendance for follow up examination and blood sampling for safety evaluation were considered unlikely. The mean age of the 113 patients was $27 \cdot 4$ (range 18-63) years and their mean weight $71 \cdot 1 \mathrm{~kg}$ (range $51-90 \mathrm{~kg}$ ). Forty nine $(43 \%)$ men were white. Sixty nine $(61 \%)$ men presented with their first attack of gonorrhoea and $44(39 \%)$ with a repeat attack (having had one to five previous episodes).

\section{BACTERIOLOGICAL TECHNIQUES}

Swabs of the urethral discharge were taken for microscopical examination of a Gram stained smear and were plated direct on to gonococcal selective media (Oxoid GC agar base CM 367, plus supplement SR 56 and haemoglobin L 53) containing vancomycin, colistin, nystatin, and trimethoprim (VCNT). All inoculated gonococcal selective media were kept at $37^{\circ} \mathrm{C}$ in the clinic incubator until at a convenient time the same day they were transferred to the $\mathrm{CO}_{2}$ incubator in the laboratory. All plates were incubated for $24-48$ hours at $37^{\circ} \mathrm{C}$ in $10 \% \mathrm{CO}_{2}$. $N$ gonorrhoeae was isolated and identified according to standard procedures. The fluorescent antibody test (BACTO-FA $N$ gonorrhoeae antiserum) and the Phadebact gonococcus test (Pharmacia, Hounslow, UK) were used to confirm the identification.

Routine sensitivity tests were performed using the controlled sensitivity disc test method. ${ }^{4}$ All strains were tested against penicillin (1 unit), ampicillin $(2 \mu \mathrm{g})$, sultamicillin (ampicillin $2 \mu \mathrm{g}+$ sulbactam $2 \mu \mathrm{g})$, and kanamycin $(30 \mu \mathrm{g})$. Strains resistant to penicillin were tested against spectinomycin $(100 \mu \mathrm{g})$, rosoxacin $(5 \mu \mathrm{g})$, and cefuroxime $(30 \mu \mathrm{g})$.

Penicillinase production was determined by the Intra-lactam test system (Diamed Diagnostics Liverpool, United Kingdom). Plasmid determinations of the $\beta$-lactamase producing strains were performed by the Venereal Disease Reference Laboratory (VDRL, London).

Minimum inhibitory concentrations (MICs) of ampicillin were determined for all strains using the plate incorporated method. ${ }^{5}$ Ampicillin concentrations ranged from 0.01 to $\geqslant 6.4 \mathrm{mg} / \mathrm{l}$. A Denley multipoint inoculator was used to transfer $1 \mu \mathrm{l}$ of each culture (inoculum size $=300$ colony forming units (cfu)) to produce a semiconfluent spot. Staphylococcus aureus (NCTC) 6571 and Escherichia coli NCTC 10418 were used as control organisms.

OTHER LABORATORY INVESTIGATIONS Complete blood cell count, urine analysis, liver (serum bilirubin concentration, serum aspartate aminotranseferase (AST) activity, and alkaline phosphatase activity) and renal (blood urea and serum creatinine concentrations) function tests were performed before treatment and at the first follow up. Routine serological tests for syphilis (the rapid plasma reagin test and the Treponema pallidum haemagglutination test) were also performed before treatment.

\section{TREATMENT AND FOLLOW UP}

All 113 patients were treated with $1 \mathrm{~g}$ of probenecid followed by $2 \mathrm{~g}$ sultamicillin as a single oral dose; one patient did not receive probenecid. The importance of a follow up examination was explained to the patients at their initial visit and reattendance was requested between two and seven days later and again within two weeks of treatment. Each patient was told the nature of his infection, asked to abstain from sexual intercourse and alcohol, and to attend for tests of cure. The patient was then interviewed by the social health worker or contact tracer. On each visit the patient was asked about his symptoms and any further sexual contact. He was re-examined, urethral swabs were taken for Gram stained smears and culture, and the urine was examined.

\section{Results}

\section{CULTURE}

Of the 113 men treated, $101(89 \%)$ attended at least one follow up examination within 14 days of treatment and $54(48 \%)$ attended a second. Six $(6 \%)$ of the 101 patients attending follow up examination were initially culture negative, although direct microscopy was positive, and one further patient did not have a culture performed after treatment. These seven patients were therefore also excluded from the efficacy analysis although any side effects were noted. Of the 94 patients available for analysis, 91 $(\mathbf{9 7 \%})$ were cured of their initial infection at their first follow up examination, which was carried out within an average of nine days (range two to 14 days) of treatment in $93(\mathbf{9 9 \%})$ patients in this group. The remaining three patients in the group had positive cultures for gonococci at the first follow up visit; two of the three admitted to further sexual exposure with consorts who were known to have been harbouring the infection during the period of re-exposure and the third denied re-exposure before the follow up visit. Of the 54 patients who attended the second follow up examination, none had a positive urethral culture for $N$ gonorrhoeae. In this latter group, 48 $(89 \%)$ were re-evaluated between seven and 20 days after treatment, the mode evaluation time for all 54 patients being 14 days after treatment. 
MINIMUM INHIBITORY CONCENTRATIONS

Table I shows the MICs of ampicillin for the initial infecting strain. In addition to the MICs for the gonococcal isolates from the patients included in the trial, the MICs for a further 226 strains collected from the clinic over the same period (from infected patients not in the trial) were measured for comparison. It is noteworthy that of the 14 strains in the trial group with an MIC $\geqslant 0.8 \mathrm{mg} / 1$ seven did not produce $\beta$-lactamase-that is, they were relatively resistant non-PPNG strains. Six of the seven patients infected with these strains were cured. The seventh patient in this group yielded an isolate with an MIC of ampicillin of $1.6 \mathrm{mg} / \mathrm{l}$ and was one of the three patients in the trial still infected at the follow up examination. In the parallel non-trial group there were 41 strains with MIC $\geqslant 0.8 \mathrm{mg} / 1$ of which 16 were $\beta$-lactamase producing (PPNG).

\section{PPNG STRAINS}

Seven patients were infected with PPNG strains, six of whom attended for follow up examination and were cured. Isolated from the six patients were three strains of the Asian type and three of the African type, each type occurring with or without transfer plasmid. Table II shows the plasmid analysis of these four different $\beta$-lactamase producing strain types and the MICs of ampicillin and sulbactam alone and of the combination at two inoculum sizes. All these strains were sensitive to spectinomycin (MIC $<30 \mathrm{mg} / \mathrm{l}$ ) and cefuroxime (MIC $<1 \mathrm{mg} / \mathrm{l}$ ). There was variable sensitivity to tetracycline (MIC $0.5-4 \mathrm{mg} / \mathrm{l}$ ) and streptomycin (MIC 10-50 mg/l). All strains were sensitive to kanamycin and rosoxacin by the disc test.

\section{FOLLOW UP}

Two of the 101 patients returning for follow up examination reported mild diarrhoea following sultamicillin treatment. No other side effects were reported. Differences between post-treatment and baseline values of laboratory safety indices were analysed using the Wilcoxon matched pairs signed ranks test. Results were assessed on up to 95 (according to safety index) of the 101 patients followed up. None of the differences between mean values before and after treatment was of clinical relevance. No important changes were found in the means of platelet numbers, the white cell differential count, the mean cell volume (MCV), AST activity, and blood urea and creatinine concentrations. Minor falls were found in the mean values for haemoglobin concentration, red and white blood cell count, total bilirubin concentration, and alkaline phosphatase activity.

\section{Discussion}

Although for several years many isolates of $N$ gonorrhoeae have shown relative resistance to penicillin, the first report of PPNG did not occur until $1976 .^{6}$ An earlier report had compared the in vitro activity

TABLE I Minimum inhibitory concentrations of ampicillin for trial and parallel non-trial strains of $N$ gonorrhoeae

\begin{tabular}{|c|c|c|c|c|c|c|c|c|c|c|}
\hline \multirow[b]{2}{*}{ Strains (No) } & \multicolumn{10}{|c|}{ No of strains inhibited by the following concentrations $(\mathrm{mg} / \mathrm{l})$ of ampicillin: } \\
\hline & 0.01 & 0.02 & 0.05 & $0 \cdot 1$ & $0 \cdot 2$ & 0.4 & $0 \cdot 8$ & $1 \cdot 6$ & $3 \cdot 2$ & $\geqslant 6 \cdot 4$ \\
\hline Trial strains (102) & 2 & 12 & 26 & 15 & 17 & 16 & 5 & 2 & $\mathbf{0}$ & $7^{*}$ \\
\hline Parallel non-trial strains (226) & 3 & 15 & 62 & 48 & 27 & 30 & 13 & 10 & 2 & $16^{*}$ \\
\hline
\end{tabular}

*All strains produced $\beta$-lactamase.

TABLE II Minimum inhibitory concentrations of ampicillin, sulbactam, and the combination (sultamicillin) for four different $\beta$-lactamase producing strains of $N$ gonorrhoeae

\begin{tabular}{|c|c|c|c|c|c|}
\hline \multirow[b]{2}{*}{ Strain type } & \multirow{2}{*}{$\begin{array}{l}\text { Plasmid content } \\
\text { (megadaltons) }\end{array}$} & \multirow{2}{*}{$\begin{array}{l}\text { Inoculum } \\
\text { concentrution }\end{array}$} & \multicolumn{3}{|c|}{$M I C(\mathrm{mg} / \mathrm{l})$ of: } \\
\hline & & & Ampicillin & Sulbactam & Sultamicillin \\
\hline $\begin{array}{l}\text { Asian } \\
\text { Asian with } \\
\text { transfer } \\
\text { plasmid }\end{array}$ & $\begin{array}{l}2 \cdot 6,4 \cdot 4 \\
2 \cdot 6,4 \cdot 4,24 \cdot 5\end{array}$ & $\begin{array}{l}1 \text { in } 10 \\
1 \text { in } 100 \\
1 \text { in } 10 \\
1 \text { in } 100\end{array}$ & $\begin{array}{l}25 \cdot 0 \\
12 \cdot 5 \\
25 \cdot 0 \\
12 \cdot 5\end{array}$ & $\begin{array}{l}3 \cdot 12 \\
3 \cdot 12 \\
3 \cdot 12 \\
3 \cdot 12\end{array}$ & $\begin{array}{l}0 \cdot 78 \\
0 \cdot 78 \\
0 \cdot 78 \\
0 \cdot 78\end{array}$ \\
\hline
\end{tabular}

*Dilutions of overnight cultures of $10^{6}-10^{7}$ colony forming units. 
of 12 antibacterial agents against $N$ gonorrhoeae in anticipation of forthcoming therapeutic difficulties. ${ }^{7}$ Many alternatives to the penicillins have been tried in the treatment of gonorrhoea but the penicillins approximate to the ideal antigonococcal agents provided that the patient is not hypersensitive to penicillin.

The effective dose of penicillin has, however, been increased step by step at regular intervals during the past few years to a level at which patient tolerance is seriously questioned. Whereas parenteral penicillin has been advocated for several years for many good reasons, recent practice has been to give a single supervised dose of a penicillin with probenecid by mouth. In 1972 the most satisfactory single dose oral treatment for gonorrhoea in both sexes was ampicillin $2 \mathrm{~g}$ with $1 \mathrm{~g}$ probenecid. ${ }^{8}$ This treatment was simple, easy to administer, acceptable to most patients, and produced cure rates of over $95 \%$. At present the practice in the United Kingdom varies, 9 and the recommended dose in the United States has risen to $3.5 \mathrm{~g}$ ampicillin with $1 \mathrm{~g}$ probenecid. ${ }^{10}$ This regimen gives a cure rate of $92-96 \%$. Successful results have been recorded with amoxycillin alone and with probenecid, as well as with the ampicillin prodrugs talampicillin and bacampicillin. None of these penicillin regimens with or without probenecid has any effect, however, against the PPNG strains which have been isolated increasingly in the United Kingdom since $1976 .{ }^{311}$ There were 115 reports of PPNG isolation to the Communicable Disease Surveillance Centre (CDSC) and the Venereal Disease Reference Laboratory (VDRL) in July-December 1980. In the same period in 1981 reports of PPNG isolation had risen to 247 while those for the first six months of 1982 increased to 420 .

PPNG strains are spreading from West Africa and the Far East to the United Kingdom. In August 1981 Jephcott et al showed that plasmid types corresponded with the source country. ${ }^{12}$ In October 1980 van Embden et al identified in the Netherlands a new plasmid combination of African type isolates comprising the 3.2 megadalton $\beta$-lactamase encoding plasmid and a 24.5 megadalton transfer plasmid. ${ }^{13}$ Since then further reports have confirmed that genetic interchange between gonococci is occur ring. ${ }^{14-16}$ In this study seven PPNG strains were isolated, one of which showed the African $\beta$-lactamase type with the additional conjugative plasmid. The plasmid analysis is shown in table II together with the MICs to ampicillin, sulbactam, and the combination (sultamicillin). The synergistic effect of ampicillin plus sulbactam for the four different PPNG strains is clearly shown.

The discovery of PPNG strains may change the therapeutic approach to the treatment of gonorrhoea since there is no way of predicting whether an infecting strain produces $\beta$-lactamase or not. Alternative regimens have been sought, hastened by early reports of spectinomycin resistant PPNG. ${ }^{17}$ Slack et al suggested treatment with cefotaxime,$^{18}$ while Tupasi et al had success with oral cefaclor. ${ }^{19}$ For disseminated infection caused by PPNG the Centers for Disease Control now recommend treatment with cefoxitin $1.0 \mathrm{~g}$ or cefotaxime $0.5 \mathrm{~g}$ four times a day intravenously for at least seven days. ${ }^{20}$

In this study we examined the clinical efficacy of sultamicillin (a prodrug delivering sulbactam, a potent $\beta$-lactamase inhibitor, plus ampicillin) and probenecid in the treatment of 113 cases of uncomplicated gonococcal urethritis in men. Seven of the patients were infected with PPNG strains. Of the 94 patients meeting the criteria for analysis at the first follow up examination, $91(97 \%)$ including six with PPNG strains were cleared of infection. Two $(3 \%)$ of the remaining three patients admitted to further sexual contact with untreated partners, and the evidence from contact tracing supports the view that these cases were probable reinfections. The third patient denied further sexual intercourse and was thus deemed to be a treatment failure. Clinically relevant side effects of sultamicillin were not evident and patient compliance was good.

In addition to the appearance of PPNG strains there has been a gradual increase in penicillin resistance during the 1970s. In 1980 Shtibel reported two strains with MICs of ampicillin $>1 \mathrm{mg} / \mathrm{l} .{ }^{21}$ In our study five strains had MICs of ampicillin of $0.8 \mathrm{mg} / 1$ and two MICs of ampicillin of $1.6 \mathrm{mg} / \mathrm{l}$; all seven were non-PPNG strains. Six of the patients infected with these strains were cured at the first follow up examination and the seventh, with an MIC of ampicillin of $1.6 \mathrm{mg} / \mathrm{l}$, was the only clear failure in the trial.

On the basis of this evidence it would seem that sultamicillin is a welcome and novel innovation in the oral treatment of non-PPNG strains with high and low MICs of ampicillin and also of PPNG strains.

We thank Dr D Geraint James and Dr A Boulton Hewitt for their support, the staff of the departments of genitourinary medicine and microbiology, and Dr N Johnston of the Venereal Disease Reference Laboratory (London) for the plasmid analysis. We thank Pfizer Central Research for the generous supply of sultamicillin and Dr David Cox for his kind advice and support.

\section{References}

1. Cox DA, Lees LJ, de Palol J, Wharfe SMW. The bioavailabilities of sulbactam and ampicillin and dose response following oral administration of sultamicillin in single doses of 250, 500, and $750 \mathrm{mg}$. In: Proceedings of the twenty-second Interscience Conference on Antimicrobial Agents and Chemotherapy. 1982; Abstract 518. 
2. English AR, Retsema JA, Girard AE, Lynch JE, Barth WE. CP-45, 899, a beta-lactamase inhibitor that extends the antibacterial spectrum of beta-lactams: initial bacteriological characterisation, Antimicrob Agents Chemother 1978; 14: 414-9.

3. McCutchan JA, Adler MW, Berrie JRH. Penicillinaseproducing Neisseria gonorrhoeae in Great Britain, 1977-81: alarming increase in incidence and recent development of endemic transmission. $B r$ Med $J$ 1982; 285: 337-40.

4. Stokes EJ. Clinical bacteriology. 3rd ed. London: Edward Arnold Ltd, 1968: 170-216.

5. Bennet JV, Brodie JL, Kirby WMM. Simplified, accurate method for antibiotic assay of clinical specimens. Applied Microbiology 1966;14:176-7.

6. Phillips I. Beta-lactamase-producing penicillin-resistant gonococcus. Lancet 1976; ii:656-7.

7. Phillips I, Ridley M, Rimmer D, Lynn R, Warren C. In vitro activity of twelve antibacterial agents against Neisseria gonorrhoeae. Lancet 1970; i:263-5.

8. Anonymous. Single-dose treatment of gonorrhoea [Editorial]. Lancet 1972; i: 885-6.

9. Adler MW. Diagnostic, treatment, and reporting criteria for gonorrhoea in sexually transmitted disease clinics in England and Wales. Br J Vener Dis 1978;54:15-23.

10. Centers for Disease Control. Gonorrhea: CDC recommended treatment schedules 1979. Sex Transm Dis 1979;6 suppl:89-92.

11. Percival A, Corkill JE, Arya OP, et al. Penicillinase-producing gonococci in Liverpool. Lancet 1976; ii: 1379-82.

12. Jephcott AE, Dickgiesser N, McClean AN. Penicillinaseproducing gonococci in Britain. Lancet 1981;ii:247-8.
13. van Embden JDA, van Klingeren $B$, Dessens-Kroon $M$, van Wijngaarden LJ. Emergence in the Netherlands of penicillinase-producing gonococci carrying "Africa" plasmids in combination with transfer plasmid. Lancet $1981 ; \mathrm{i}: 938$.

14. Ansink-Schipper MC, van Embden JDA, van Klingeren B, Woudstra R. Further spread of plasmids among different auxotypes of penicillinase-producing gonococci. Lancet 1982; i: 445 .

15. Johnston NA, Kolator B. Emergence in Britain of betalactamase producing gonococci with new plasmid combination. Lancet 1982; i: 445-6.

16. Jephcott AE, Egglestone SI, Copley C. Further evidence of dissemination of ability to produce penicillinase amongst gonococci. Lancet 1982; i: 1467-8.

17. Ashford WA, Potts DW, Adams HJU, et al. Spectinomycinresistant penicillinase-producing Neisseria gonorrhoeae. Lancet 1981; ii: 1035-7.

18. Slack RCB, Bittiner JB, Finch R. Treatment of gonorrhoea caused by beta-lactamase producing strain of Neisseria gonorrhoeae with cefotaxime. Lancet 1980; i:431-2.

19. Tupasi TE, Calubiran OV, Torres CA. Single oral dose of cefaclor for the treatment of infections with penicillinaseproducing strains of Neisseria gonorrhoeae. Br J Vener Dis 1982; 58: 176-9.

20. Centers for Disease Control. Sexually transmitted diseases: treatment guidelines, 1982. Morbidity and Mortality Weekly Report 1982; 32: 405 .

21. Shtibel R. Non-beta-lactamase producing Neisseria gonorrhoeae highly resistant to penicillin. Lancet 1980; ii: 39 . 